\title{
Los cromosomas mitóticos y meióticos del pez tropical Petenia splendida (Cichlidae)
}

\author{
Lenin Arias-Rodriguez ${ }^{1}$, Leonardo Ibarra-Castro ${ }^{2} \&$ Salomón Páramo-Delgadillo $^{1}$ \\ 1. División Académica de Ciencias Biológicas, UJAT, 967 Villahermosa, Tabasco, México; \\ leninariasrodriguez@hotmail.com \\ 2. Centro de Investigación en Alimentación y Desarrollo, Unidad Mazatlán, Sábalo-Cerritos S/N, Estero del Yugo, 711 \\ Mazatlán, Sinaloa, México.
}

Recibido 18-V-2006. Corregido 25-X-2007. Aceptado 26-XI-2007.

\begin{abstract}
The mitotic and meiotic chromosomes of the tropical fish Petenia splendida (Cichlidae). The karyotype of bay snook, Petenia splendida, is described based on mitotic and meiotic stages of sixty larvae and twelve juveniles from Tabasco, Mexico. Standard cytological procedures with minor modifications were followed to obtain mitotic and meiotic chromosome spreads. One hundred chromosome slides were analyzed and 290 chromosome spreads were counted. High-quality spreads in mitosis and meiosis were used for karyotype analysis. Mitotic chromosome spreads showed $76.7 \%$ of such cells with $2 \mathrm{n}=48$ chromosomes, while meiotic spreads revealed $55.2 \%$ with 24 chromosomes in haploid stage. Photographic documentation of eight highquality pictures showed that the karyotype consists of three pairs of bi-armed metacentric-submetacentric chromosomes (msm) and 21 pairs with uni-armed subtelocentric-acrocentric chromosomes (sta), with a fundamental number (FN) of 54 arms. Karyotype chromosomes were verified by analysis of haploid and diploid metaphases at meiotic stage I. Abundant chromosome spreads were observed more frequently on slides from larvae. No evidence of heteromorphism to discriminate sexual chromosomes was detected. There were "dot-like" chromatic bodies in both sexes and they were classified as "B" chromosomes. The karyotype of $P$. splendida is type "A", i.e. primitive in the Cichlid family, similar to other species of Cichlasoma. The occurrence of supernumerary chromosomes is still unknown: studies on the effects of pollution and hybridization might be important to understand that phenomenon. Rev. Biol. Trop. 56 (2): 895-907. Epub 2008 June 30.
\end{abstract}

Key words: Tenguayaca, Petenia splendida, B chromosomes, karyotype, cichlid.

La mojarra tenguayaca es un cíclido neotropical que habita en la cuenca del río GrijalvaUsumacinta (Miller 1992), cuyo ámbito de distribución comprende cuerpos de agua continentales y salobres (Miller 1992, EspinosaPérez 1993). Por su introducción en la presa de Temascal, Oaxaca, también está presente en el río Papaloapan, en el sur de México (EspinosaPérez 1993). En México es un recurso de subsistencia para las comunidades ribereñas, junto con otras especies dulceacuícolas. Debido a la sobrepesca y el incremento en los índices de contaminación, se han investigado especies nativas (p.e. la tenguayaca) que presentan características adecuadas para cultivo como alternativa a la importación de especies exóticas como las tilapias (Dunseth y Bayne 1978, Martínez-Palacios y Ross 1988, Mendoza et al. 1992, Mendoza et al. 1995, Pérez-Sánchez et al. 2005).

Siendo representante monoespecífico del género Petenia, es una especie que ha despertado interés para la comparación de varios procesos biológicos relacionados con los cíclidos Mesoamericanos. El aspecto trófico es uno de los más estudiados, principalmente por las características particulares de tamaño y morfología que muestra la boca antes y después de la captura de una presa. Dichas características han conferido a la especie mayor 
capacidad y plasticidad durante la captura de una gran variedad de presas en movimiento (Waltzek y Wainwright 2003, Hulsey y García de León 2005). La predominancia del hábito ictiófago ha sido sugerida solo para los jóvenes y adultos o especímenes de talla moderada y del habito zooplanctófago solo en los estadios de larva-juvenil (Reséndez-Medina y Salvadores-Baledón 1983, Parámo-Delgadillo 1984, Chávez et al. 1988, Valtierra-Vega y Schmitter-Soto 2000).

La especialización bucal y alimenticia mostrada por varias especies de cíclidos Mesoamericanos de acuerdo con Hulsey y García de León (2005) han sido mecanismos importantes para la diversificación de estas especies. En el caso de P. splendida, la flexibilidad que muestra la boca da mayores posibilidades de adaptación y diversificación (Hulsey y García de León 2005); lo que se refleja por su presencia en ambientes diversos (GamboaPérez y Schmitter-Soto 1998, Valtierra-Vega y Schmitter-Soto 2000).

La plasticidad para adaptarse, sugiere la existencia de variación genética a nivel específico como ha sido demostrado en el complejo de especies de la mojarra común que habitan en dos lagos de Nicaragua (McKaye et al. 2002). Por lo que en América se han reconocido varias especies de mojarras y estas comprenden grupos monofiléticos en el cual deberían estar consideradas especies de Centroamérica y Sudamérica (Hulsey et al. 2004). Dicha información contrasta con las evidencias citogenéticas que indican un número cromosómico ancestral de 48 cromosomas en la mayoría de los cíclidos Americanos, por lo que se considera como un grupo de peces con evolución cariotípica conservada (Gyldenholm y Scheel 1971, Thompson 1979, Rab et al. 1983, Feldberg y Bertollo 1984, 1985a, b, Salas y Boza 1991, Martins et al. 1995, Uribe-Alcocer et al. 1992, 1999, Arias-Rodriguez et al. 2006).

En el presente documento se establece por primera ocasión el cariotipo de $P$. splendida a partir del análisis del complemento cromosómico en mitosis y meiosis.

\section{MATERIALES Y MÉTODOS}

Procedimiento citogenético: se procesaron 60 larvas de seis días post-eclosión, provenientes de la reproducción en cautiverio y 12 juveniles (seis hembras y seis machos) procedentes de las lagunas adyacentes a la ciudad de Villahermosa, Tabasco en México. El sexo de los especímenes fue determinado por el procedimiento recomendado por ParámoDelgadillo (1985).

En las larvas y juveniles se siguieron con algunas modificaciones, los procedimientos citológicos de Denton (1973) y de Kligerman y Bloom (1977) como se señala a continuación.

Las larvas fueron mantenidas en una solución de colchicina al $0.005 \%$ por $12 \mathrm{~h}$ y a los jóvenes se les inyectó colchicina que fue disuelta en una solución de citrato de sodio al $0.1 \%$ a una concentración de $28 \mu \mathrm{g} / \mathrm{g}$ de peso del ejemplar y exposición de 6 hrs. Las larvas y los tejidos (branquias, riñón y gónadas) fueron hidratados durante $80 \mathrm{~min}$ en una solución de citrato de sodio al $1.0 \%$, y fijados posteriormente con metanol $\left(4{ }^{\circ} \mathrm{C}\right)$ y ácido acético en proporción $4: 1$. El fijador se reemplazó cada $10 \mathrm{~min}$ por centrifugación a 5000 r.p.m en cuatro ocasiones. Los tejidos fueron preservados a $-20^{\circ} \mathrm{C}$.

El tejido fijado, se utilizó para preparar las laminillas por la técnica de goteo desde una altura aproximada de $1.70 \mathrm{~m}$ y secado a la flama con un mechero de alcohol. Inmediatamente, las laminillas fueron incubadas $24 \mathrm{hrs}$ a $45^{\circ} \mathrm{C}$ y a continuación fueron teñidas durante $15 \mathrm{~min}$ en una solución de giemsa al $10 \%$ preparada en fosfato buffer a $\mathrm{pH}=7.0$.

Los organismos sacrificados fueron preservados para su determinación taxonómica de acuerdo con la descripción original de Günther (1867) y finalmente fueron depositados en la colección Nacional de Peces de la Universidad Nacional Autónoma de México, con número de registro: 14434 IBUNAM-P.

Elaboración del cariotipo y análisis: se analizaron 100 laminillas para localizar dispersiones cromosómicas adecuadas en mitosis 
$\mathrm{y}$ meiosis, con los objetivos 10X, 40X y 100X de un microscopio Olimpus CX-31. Las mejores dispersiones fueron digitalizadas con un microscopio DMLB y cámara MPS 30 (Leyca).

El número cromosómico modal diploide fue determinado por conteo de los cromosomas presentes en las mejores dispersiones cromosómicas en impresiones de alta resolución.

En la elaboración del cariotipo, se ampliarón y recortarón ocho (cinco de machos y tres de hembras) de los mejores campos mitóticos de los jóvenes. Los cromosomas fueron colocados en orden de longitud descendente y de acuerdo a la posición del centrómero. Se tomaron las medidas de longitud total de los brazos p (brazo corto) y q (brazo largo) de cada cromosoma, se calculó el valor medio y la desviación estándar de cada par homólogo de cromosomas. Se clasificaron los cromosomas en acuerdo con el criterio de Levan et al. (1964): longitud relativa de cada par de cromosomas [longitud del cromosoma $\mathrm{p}+\mathrm{q} /$ longitud total del complemento cromosómico haploide (100)], proporción de brazos $(\mathrm{r}=\mathrm{q} / \mathrm{p})$, índice centromérico $[\mathrm{I} . \mathrm{C}=100(\mathrm{p} / \mathrm{p}+\mathrm{q})]$ y la diferencia entre brazos $[\mathrm{d}=\mathrm{r}-1(10) / \mathrm{r}+1]$.

El número fundamental (NF), fue establecido de acuerdo al número de brazos cromosómicos y el ideograma fue construido en orden decreciente de longitud relativa de cada par homólogo de cromosomas (Denton 1973).

\section{RESULTADOS}

Se contabilizaron 176 campos mitóticos de los cuales $76.7 \%$ corresponde a una moda de $2 \mathrm{n}=48$ cromosomas (Fig. 4B, D), mientras en 114 campos cromosómicos en meiosis, el 55.2 $\%$ correspondió a una moda de $1 \mathrm{n}=24$ cromosomas (Fig. 1A, 2, 4C). En los conteos previos, se identificó un campo pentaploide con $5 \mathrm{n}=120$ cromosomas (Fig. 4A).

En las dispersiones cromosómicas seleccionadas para establecer el cariotipo, se identificaron tres pares como cromosomas metacéntricos-submetacéntricos (msm) y 21 pares como subtelocéntricos-acrocéntricos (sta) (Fig. 1B, 2A, Cuadro 1).

La presencia de cromosomas msm y sta en el cariotipo de $P$. splendida, fue verificada por el análisis de una dispersión cromósomica en meiosis (I) de la profase de una célula espermática en estadio cigoteno (Fig. 3). Las dispersiones cromosómicas de las larvas fueron mas abundantes en comparación con la mostrada por los especímenes en estadio juvenil (Fig. 4F).

No fue posible identificar diferencias heteromórficas que correspondan a cromosomas sexuales (Fig. 2, 3 y 4). Sin embargo, se logró observar en las muestras de gónadas y branquias de tres hembras y dos machos la presencia de uno a cinco cuerpos cromáticos en forma de punto, adicionales al complemento cromosómico y que son característica particular de los denominados microcromosomas tipo "B" (Fig. 5).

\section{DISCUSIÓN}

El cariotipo de $P$. splendida, está formado por seis cromosomas metacéntricossubmetacéntricos (birrámeos), 42 cromosomas subtelocéntricos-acrocéntricos (unirrámeos) y con número fundamental de 54 brazos cromosómicos. La comparación de los resultados de Thompson (1979) con los de P. splendida la ubica como una especie citogenéticamente muy cercana a los miembros del género Cichlasoma de Günther (1867).

En aproximadamente 100 estudios citogenéticos realizados en alrededor de 200 especies de cíclidos neotropicales, los resultados muestran como número modal diploide 48 elementos cromosómicos en su mayoría del tipo subtelocéntricos-telocéntricos (stt) (Gyldenholm y Scheel 1971, Thompson 1979, Rab et al. 1983, Feldberg y Bertollo 1984, 1985a, b, Salas y Boza 1991, Martins et al. 1995, UribeAlcocer et al. 1992, 1999, Arias-Rodriguez et al. 2006).

La presencia de 48 cromosomas en condición diploide es un carácter citotaxonómico 

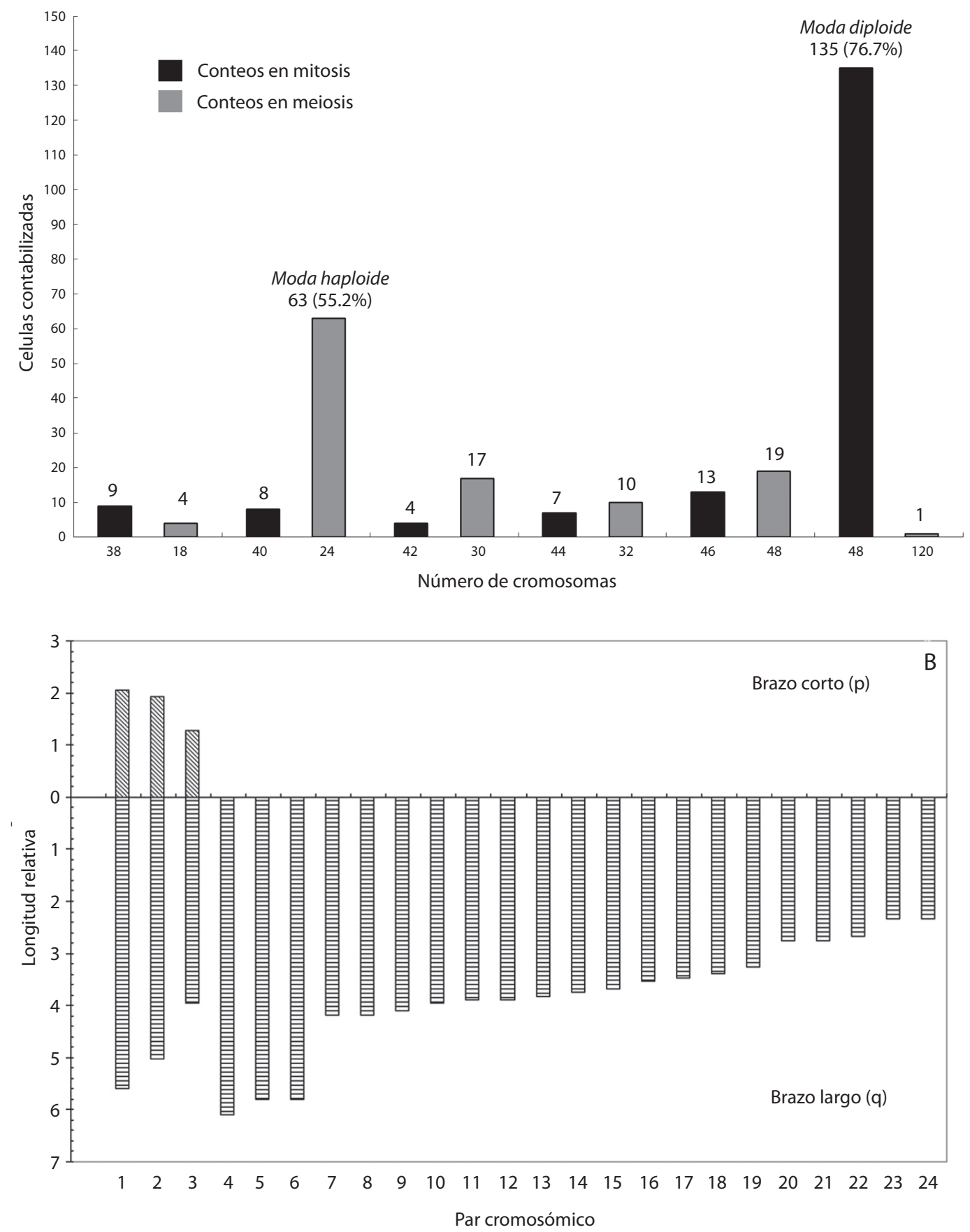

Fig. 1. (A) Frecuencia del número cromosómico y número modal haploide y diploide; y (B) el idiograma representativo del complemento cromosómico promedio por pares del cariotipo de P. splendida.

Fig. 1. (A) Frequency of chromosomes, haploid and diploid modal numbers; and (B) the representative idiogram from the mean chromosome pairs complement of the P. splendida karyotype. 


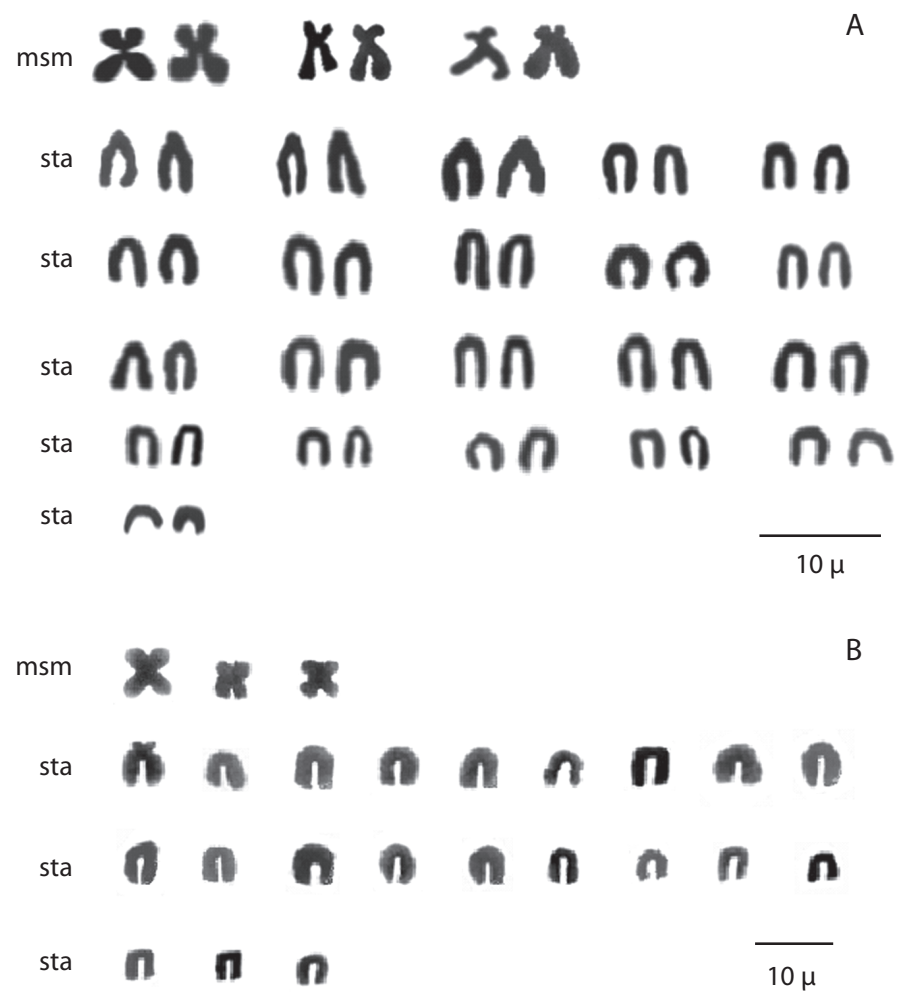

Fig. 2. (A) Cariotipo representativo de la mojarra tenguayaca P. splendida $2 \mathrm{n}=48$; (B) los cromosomas en meiosis I del cariotipo haploide $1 \mathrm{n}=24$.

Fig. 2. (A) Representative karyotype of the Bay Snook P. splendida $2 \mathrm{n}=48$; (B) and the chromosomes in meiosis I from the haploid karyotype $1 \mathrm{n}=24$.

común en la gran mayoría de los cíclidos de América, hecho que coincide con los resultados del número modal en meiosis $(1 \mathrm{n}=24)$ y mitosis $(2 \mathrm{n}=48)$ en $P$. splendida (Fig. 1A, 2, 4B-E). Dicho número, se considera ancestral en los cíclidos americanos por lo que se distingue como un grupo de peces con evolución cariotípica conservada (Nishikawa et al. 1973, Thompson 1979, Rab et al. 1983, Feldberg y Bertollo 1984, 1985a, b, Salas y Boza 1991, Martins et al. 1995, Uribe-Alcocer et al. 1992, 1999, Arias-Rodriguez et al. 2006).

Sin embargo, los valores del número fundamental (NF) en apariencia son específicos para cada género; por ejemplo en Cichlasoma, varía de 52 a 60 brazos cromosómicos (Thompson
1979, Martins et al. 1995), con una excepción notoria en $C$. salvini $\mathrm{NF}=80$ (Thompson 1979), mientras que en Apistogramma va de 60-70, en Geophagus es de NF=50-70, en Crenicichla el NF=54-66, en Nannacara es $\mathrm{NF}=62$ y en Symphysodon NF=118. De acuerdo a Thompson (1979) dichas tendencias no tienen relación directa con el estatus genérico.

Las diferencias en el NF, esta relacionada con el criterio de clasificación de los cromosomas principalmente aquellos conocidos como monorrámeos. Los cromosomas monorrámeos se observan con frecuencia en los cariotipos de los cíclidos centroamericanos y estos muestran en muchos casos la presencia de brazos pequeños que se ubican por encima del centrómero. 


\section{CUADRO 1}

Longitudes promedio relativas ( \pm D.E.) de ocho cariotipos procedentes de cinco machos y tres hembras y análisis del complemento cromosómico diploide de $\mathrm{P}$. splendida

TABLE 1

Mean relative lengths $( \pm$ S.E) of eight karyotypes from five males and three females and analysis of the diploid chromosome complement for P. splendida

\begin{tabular}{|c|c|c|c|c|c|c|c|}
\hline $\begin{array}{c}\text { Número } \\
\text { de par } \\
\text { cromosómico }\end{array}$ & $\begin{array}{l}\text { Longitud } \\
\text { relativa } \\
\mathrm{p} \pm \text { D.E }\end{array}$ & $\begin{array}{l}\text { Longitud } \\
\text { relativa } \\
\mathrm{q} \pm \mathrm{D} . \mathrm{E}\end{array}$ & $\begin{array}{l}\text { Longitud } \\
\text { relativa } \\
\mathrm{p}+\mathrm{q}\end{array}$ & $\mathrm{r}$ & $\mathrm{i}$ & $\mathrm{d}$ & Clasificació \\
\hline 1 & $5.62 \pm 0.78$ & $2.06 \pm 0.26$ & 7.68 & 2.72 & 26.8 & 4.63 & $\mathrm{msm}$ \\
\hline 2 & $5.05 \pm 0.50$ & $1.92 \pm 0.53$ & 6.97 & 2.63 & 27.5 & 4.49 & $\mathrm{msm}$ \\
\hline 3 & $3.98 \pm 0.60$ & $1.28 \pm 0.23$ & 5.26 & 3.11 & 24.3 & 5.14 & $\mathrm{msm}$ \\
\hline 4 & $6.12 \pm 0.44$ & & 6.12 & & & & sta \\
\hline 5 & $5.83 \pm 0.74$ & & 5.83 & & & & sta \\
\hline 6 & $5.83 \pm 0.44$ & & 5.83 & & & & sta \\
\hline 7 & $4.20 \pm 0.70$ & & 4.20 & & & & sta \\
\hline 8 & $4.20 \pm 0.59$ & & 4.20 & & & & sta \\
\hline 9 & $4.13 \pm 0.35$ & & 4.13 & & & & sta \\
\hline 10 & $3.98 \pm 0.53$ & & 3.98 & & & & sta \\
\hline 11 & $3.91 \pm 0.42$ & & 3.91 & & & & sta \\
\hline 12 & $3.91 \pm 0.50$ & & 3.91 & & & & sta \\
\hline 13 & $3.84 \pm 0.44$ & & 3.84 & & & & sta \\
\hline 14 & $3.77 \pm 0.26$ & & 3.77 & & & & sta \\
\hline 15 & $3.70 \pm 0.46$ & & 3.70 & & & & sta \\
\hline 16 & $3.56 \pm 0.23$ & & 3.56 & & & & sta \\
\hline 17 & $3.49 \pm 0.82$ & & 3.49 & & & & sta \\
\hline 18 & $3.41 \pm 0.38$ & & 3.41 & & & & sta \\
\hline 19 & $3.27 \pm 0.52$ & & 3.27 & & & & sta \\
\hline 20 & $2.77 \pm 0.15$ & & 2.77 & & & & sta \\
\hline 21 & $2.77 \pm 0.90$ & & 2.77 & & & & sta \\
\hline 22 & $2.70 \pm 0.35$ & & 2.70 & & & & sta \\
\hline 23 & $2.35 \pm 0.90$ & & 2.35 & & & & sta \\
\hline 24 & $2.35 \pm 0.18$ & & 2.35 & & & & sta \\
\hline
\end{tabular}

D.E = desviación estándar; $\mathrm{p}=$ brazo corto $\mathrm{q}=$ brazo largo $; \mathrm{r}=$ proporción de brazos; $\mathrm{i}=$ índice centromérico; $\mathrm{d}=$ diferencia entre brazos; $\mathrm{msm}=$ metacéntrico-submetacéntrico; sta $=$ subtelecéntrico-acrocéntrico 


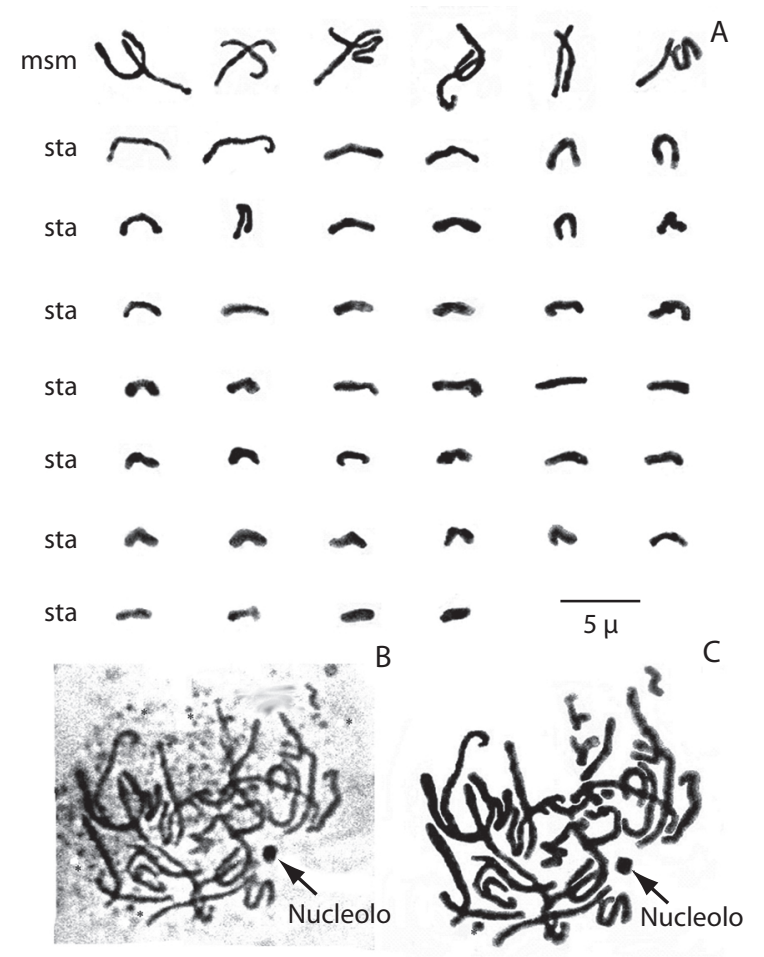

Fig. 3. (A) Cariotipo con 48 cromosomas meióticos; (B) profase de una célula espermática en estadio cigoteno; (C) campo meiótico retocada digitalmente a partir de la imagen B; por último el seccionado de cromosomas como se muestran en (A).

Fig. 3. (A) Karyotype with 48 meiotic chromosomes; (B) spermatic cell at prophase in zygotene stage; (C) meiotic spread refined electronically from (B); afterwards the chromosomes cut off as in (A).

Las dificultades de medición, hicieron de la estructura un problema técnico para clasificar los cromosomas (Levan et al. 1964). No obstante, tal dificultad se elimina cuando se clasifican en una categoría intermedia tal y a como fue utilizada por Thompson (1979). Sin embargo, en algunos reportes como el de Feldberg et al. (1985a) y Martins et al. (1995), los cromosomas continúan clasificándose en categorías individuales. Aunque la morfología de algunos de los pares cromosómicos monorrámeos muestra una prolongación pequeña, por encima del centrómero, que los ubicaría técnicamente en una categoría intermedia y por lo tanto el NF podría variar si tales cromosomas escalan la categoría hacia birrameo.

En P. splendida se adoptó la clasificación de categorías intermedias (msm y sta) considerando la morfología, tamaño de los cromosomas (Fig. 1-4), los efectos de la colchicina sobre la constricción de los cromosomas, la acción de los fijadores, el tamaño de los cromosomas al momento de ser fijados (estadio de división celular) y el criterio subjetivo para la clasificación. Tales parámetros, han sido documentados como fuente importante de variación que afecta las comparaciones (Arias-Rodriguez et al. 2006); por ejemplo, las fotografías de la figura 4 , muestran los cromosomas con grados variables de constricción producto de uno o varios de los factores antes señalados. Al parecer la colchicina y los fijadores tienen mayor efecto sobre la morfología cromósomica de organismos en estadio larvario (Fig. 4F). Sin embargo, el estadio y tipo de división celular evidentemente podrían afectar las comparaciones. 

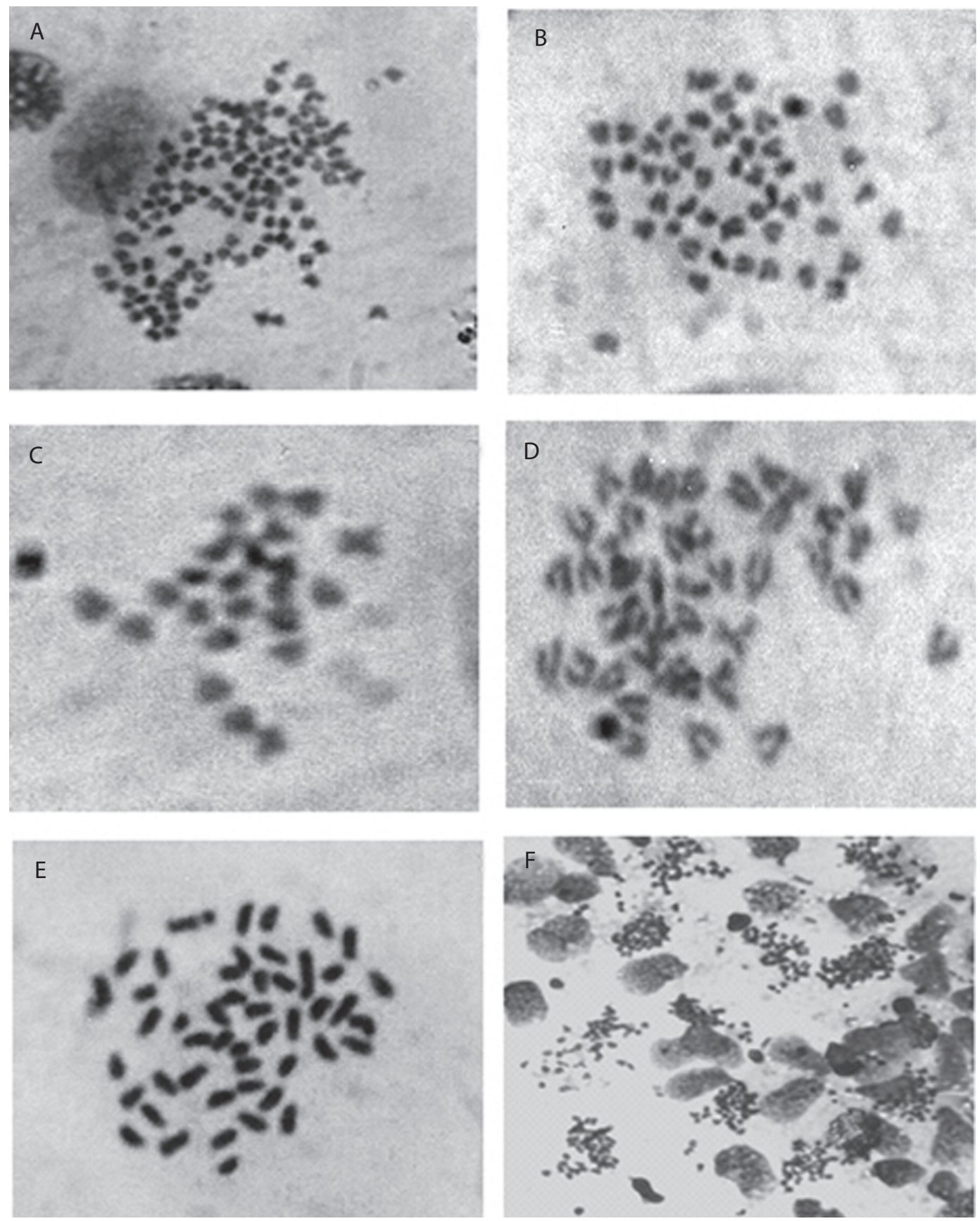

Fig. 4. (A) Cromosomas de una célula espermática en estadio leptoteno $(5 \mathrm{n}=120)$; $(\mathbf{B})$ metafase de una espermatogonia $(2 \mathrm{n}=48) ;(\mathbf{C})$ metafase I haploide con 24 bivalentes; $(\mathbf{D}, \mathbf{E})$ corresponden a cromosomas mitóticos $(2 \mathrm{n}=48) ; \mathrm{y}(\mathbf{F})$ muestra campos mitóticos $(2 \mathrm{n}=48)$ provenientes de larvas de P. splendida.

Fig. 4. (A) Chromosomes from a spermatic cell in leptotene stage $(5 \mathrm{n}=120)$; $(\mathbf{B})$ spermatogonic metaphase $(2 \mathrm{n}=48)$; (C) haploid metaphase I with 24 bivalents; (D, E) correspond to mitotic chromosomes $(2 \mathrm{n}=48)$, and (F) illustrate mitotic chromosome spreads $(2 \mathrm{n}=48)$ from larvae of $P$. splendida. 

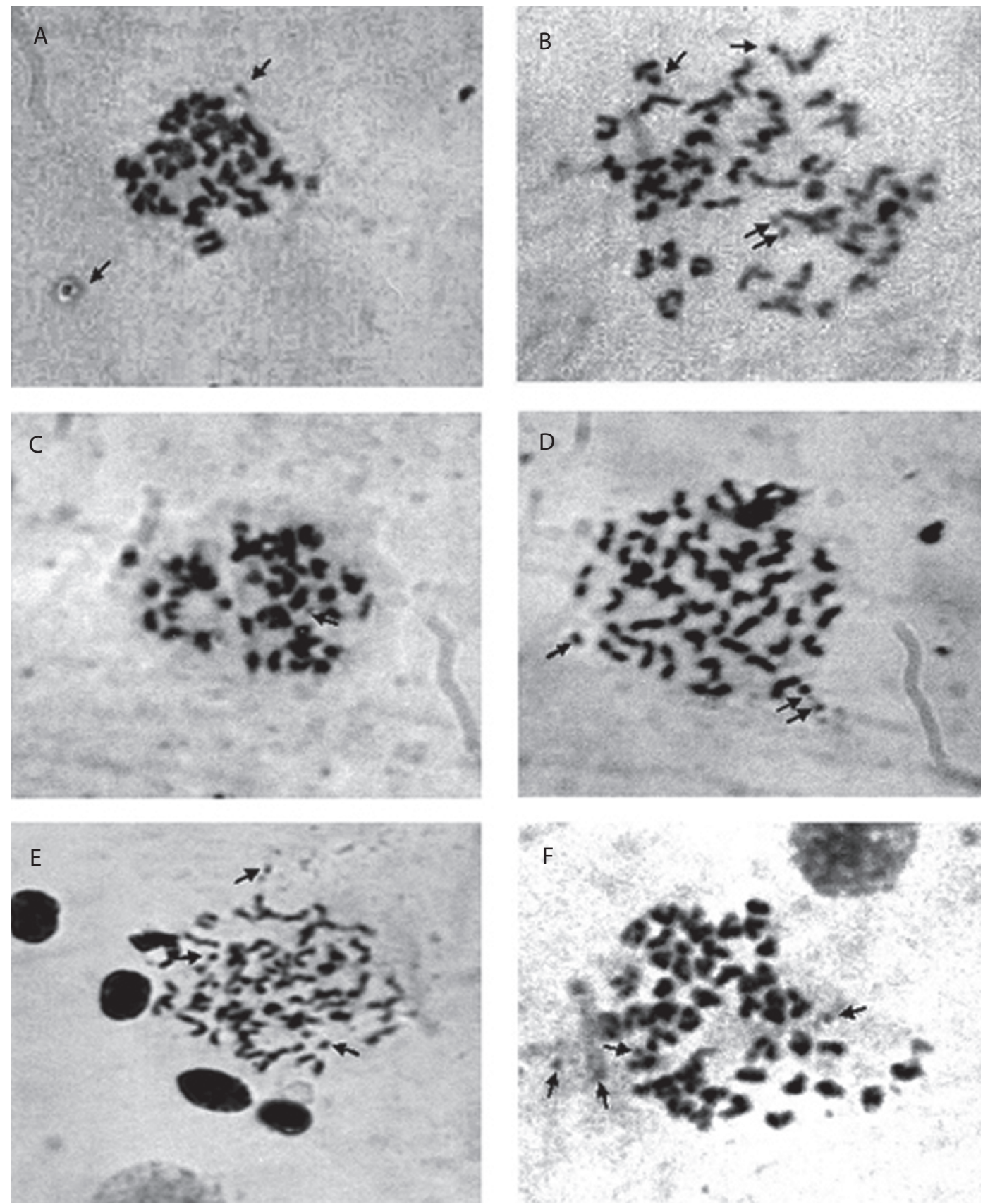

Fig. 5. (A, B) Metafases cromosómicas en meiosis; y (C, D, E y F) mitosis de P. splendida, con microcromosomas "B". Las flechas indican uno o dos microcromosomas "B", en forma de punto.

Fig. 5. (A, B) Meiotic; and (C, D, E y F) mitotic chromosome metaphases of $P$. splendida with "B" microchromosomes. Arrows point one or two "B" dot-like microchromosomes. 
En meiosis I, los cromosomas de $P$. splendida en estadio cigoteno, muestran cromosomas muy alargados (Fig. 3) y en leptoteno los cromosomas están con alto grado de constricción de la cromatina (Fig. 4A y C). Los embriones en peces, exponen cromosomas con brazos alargados debido al elevado número de divisiones celulares que no permiten la condensación adecuada de la cromatina (Thorgaard y Disney 1990). Similar al caso exhibido por las células meióticas en P. splendida, principalmente por que este evento es muy lento y da oportunidad para observar los cromosomas en diferentes estadios, aunque la cantidad de campos es muy reducida.

Por otro lado, se detectaron de una a cinco estructuras cromáticas en forma de punto, con apariencia similar a la que presentan los denominados microcromosomas o cromosomas tipo "B" y que son adicionales al complemento cromosómico estándar o cromosomas tipo "A" (Fig. 4, 5). La presencia de cromosomas supernumerarios o cromosomas " $\mathrm{B}$ " ha sido reportado en los miembros de la familia Characidae $\mathrm{y}$ en varios grupos de peces neotropicales (Portela-Castro et al. 2001). La presencia se ha observado en una o dos poblaciones de la misma especie (Moreira et al. 2001, Zhang y Arai 2003), circunstancia que indica un alto grado de diferenciación genética.

En los cíclidos sudamericanos la presencia de cromosomas "B", fue registrada por Feldberg y Bertollo (1985a) en células germinales de Gymnogeophagus balzanii. Por otro lado, Salgado et al. (1994) observaron 18 microcromosomas en Symphysondon discus. En Cichla sp., C. monoculus y Crenicichla reticulata de uno a tres microcromosomas "B", fueron identificados por Feldberg et al. (2004). También, en Geophagus surinamensis (Thompson 1979) y en G. brasiliensis, C. paranaense y C. lepidota (Martins et al. 1995), observaron la presencia de cuerpos cromáticos que bien podrían estar relacionados con la existencia de microcromosomas "B".

El origen de los microcromosomas tipo "B" en tres especies de cíclidos del Amazonas, fue ampliamente discutido por Feldberg et al.
(2004), proponiendo dos posibles escenarios, la acción mutagénica del mercurio metílico presente en el medio acuático por acción antropogénica y el origen intraespecífico debido a eventos de hibridación.

Los resultados en $P$. splendida, sugieren que la presencia de microcromosomas tipo "B" $\mathrm{y}$ cromosomas supernumerarios en meiosis (Fig. 4A, 5) puede tener un origen semejante al planteado por Feldberg et al. (2004).

Los altos índices de contaminación en los cuerpos de agua de Tabasco, originados por las actividades petroleras, el incremento de las prácticas agrícolas y antropogénicas en las márgenes de los ríos y lagunas que es el hábitat de $P$. splendida, han propiciado aumentos considerables de contaminación (Botello et al. 1983, 1991, Díaz et al. 1996) que podrían estar afectando el genoma de las especies acuáticas y en el caso de la tenguayaca se estaría observando indirectamente por presencia de microcromosomas.

Por otro lado, en la década de los 60's, se introdujeron en México varias especies de cíclidos del género Oreochromis $(2 \mathrm{n}=44)$, algunos de los cuales, pudieron escapar del cautiverio y ahora están interactuando con las especies nativas. La posibilidad de entrecruzamiento fue discutida por Martínez-Palacios y Ross (1994), pero no hay evidencias que avalen dicho fenómeno en México, aunque existe el reporte de hibridación entre $C$. monoculus y $C$. temensis especies nativas del amazonas (Alves et al. 2004).

La posibilidad de entrecruzamiento de $P$. splendida con los cíclidos africanos podría ser nula en condiciones naturales de apareamiento, debido a que el comportamiento reproductivo es diferente al igual que el número de cromosomas entre ambos grupos taxonómicos y esto corresponde a una barrera biológica para que se dé hibridación natural. Sin embargo, las similitudes de los números de cromosomas, abre la posibilidad de hacer estudios de hibridación artificial con las especies nativas y las perspectivas de estos con estudios afines de citogenética molecular, reproducción y genética que serian importantes para el buen manejo 
y aprovechamiento de este recurso, así como para el entendimiento de la biología básica de la especie.

\section{AGRADECIMIENTOS}

La presente investigación tuvo financiamiento del proyecto PROMEP(UJAT-PTC-047) para la incorporación de nuevo PTC ortogado al primer autor (L.A.R.). Se agradece la revisión realizada por dos revisores anónimos El mantenimiento de los especímenes utilizados se dio gracias a la valiosa colaboración de Rocío Chan Rodriguez.

\section{RESUMEN}

Para describir los cromosomas del cariotipo en mitosis y meiosis de la mojarra tenguayaca $P$. splendida, se procesaron 60 larvas y doce jóvenes (seis hembras y seis machos) procedentes de Tabasco, México. Se emplearon los procedimientos citológicos clásicos para peces pequeños y grandes, con algunas modificaciones que permitieron obtener campos cromosómicos en meiosis y mitosis. Analizamos al microscopio 100 laminillas, contando 290 dispersiones cromosómicas. En mitosis, $76.7 \%$ de los conteos dieron número modal diploide de $2 \mathrm{~N}=48$ cromosomas, mientras en meiosis el $55.2 \%$ mostró 24 cromosomas en condición haploide. Se analizaron ocho de las mejores fotografías para establecer el cariotipo y se identificaron tres pares de cromosomas birrámeos metacéntricos-submetacéntricos $(\mathrm{msm})$ y 21 pares de cromosomas monorrámeos subtelocéntricos-acrocéntricos (sta) con número fundamental (N.F) de 54 brazos. Se corroboró el cariotipo mediante el análisis de campos cromosómicos en estadio haploide y diploide de la meiosis I. Las dispersiones cromosómicas tuvieron un número mayor en larvas que en jóvenes. No hubo diferencias heteromórficas para distinguir cromosomas sexuales. Sin embargo, se observó la presencia de cuerpos cromáticos en forma de puntos, como una característica propia de los microcromosomas "B". Para esta familia, el cariotipo de P. splendida es primitivo o tipo "A"; y es estrechamente parecido al del género Cichlasoma. El origen de los cromosomas supernumerarios es un fenómeno aun desconocido en los cíclidos por lo que faltan estudios relacionados con el daño causado por la contaminación y la hibridación.

Palabras clave: Tenguayaca, Petenia splendida, cromosomas B, cariotipo, cíclido.

\section{REFERENCIAS}

Almeida-Toledo, L.F., F. Foresti, M.F.Z. Daniel \& S.A. Toledo-Filho. 2000. Sex chromosome evolution in fish: the formation of the neo-Y chromosome in Eigenmannia (Gymnotiformes). Chromosoma 109: 197-200.

Alves, M.N., J.I. Rebelo Porto \& E. Feldberg. 2004. Karyological evidence for interspecific hybridization between Cichla monoculus and C. temensis (Perciformes, Cichlidae) in the Amazon. Hereditas 141: 252-257.

Arias-Rodriguez, L., S. Páramo-Delgadillo \& A.L. DuránGonzález. 2006. Caracterización citogenética del pez tropical de agua dulce Parachromis managuensis (Pisces: Cichlidae). Rev. Biol. Trop. 54: 35-42.

Botello, A.V., J.A. Goñi \& S.A. Castro. 1983. Levels of organic pollution in coastal lagoons of Tabasco state, Mexico; I: Petroleum hydrocarbons. Bull. Environ. Contam. Toxicol. 3: 271-277.

Botello, A.V., C. Gonzalez \& G. Diaz. 1991. Pollution by petroleum hydrocarbons in sediments from continental shelf of Tabasco state, Mexico. Bull. Environ. Contam. Toxicol. 47: 565-571.

Chávez, L., A.E. Mattheeuws \& V.M.H. Pérez. 1988. Biología de los peces del río San Pedro en vista de determinar su potencial para la piscicultura. Inst. Nac. Invest. Recursos Bióticos, Villahermosa, México. D.F., México.

Denton, T.E. 1973. Fish chromosome methodology. Charles C. Thomas. Illinois. EEUU.

Díaz, G.G., J.C. Cámara., A.V. Botello \& G.V. Ponce. 1996. Evaluación de hidrocarburos aromáticos policíclicos en suelos hidromórficos de la reserva de la biosfera "los pantanos de Centla, en Tabasco, México. Rev. Int. Contam. Ambient. 12: 15-21.

Dunseth, D.R. \& D.R. Bayne. 1978. Recruitment control and production of Tilapia aurea (Steindachner) with the predator Cichlasoma managuense (Günther). Aquaculture 14: 383-390.

Espinoza-Pérez, H., M.T. Gaspar-Dillanes \& P. FuentesMata. 1993. Listados faunísticos de México. III. Los peces dulceacuícolas Mexicanos. Universidad Nacional Autónoma de México, Instituto de Biología, México. D.F., México.

Feldberg, E. \& L.A.C. Bertollo. 1984. Discordance in chromosome number among somatic and gonadal tissue cells of Gymnogeophagus balzanii (Pisces, Cichlidae). Braz. J. Genet. 7: 639-645. 
Feldberg, E. \& L.A.C. Bertollo. 1985a. Karyotypes of 10 species of neotropical cichlids (Pisces, Perciformes). Caryologia 38: 257-268.

Feldberg, E. \& L.A.C. Bertollo. 1985b. Nucleolar organizer regions in some species of Neotropical cichlid fish (Pisces, Perciformes). Caryologia 38: 319-324.

Feldberg, E., J.I.R. Porto, M.N. Alves-Brinn, M.N.C. Mendonça \& D.C. Benzaquema. 2004. B chromosomes in Amazonian cichlid species. Cytogenet. Genome Res. 106: 195-198.

Gamboa-Pérez, H.C. \& J.J. Schmitter-Soto. 1998. Distribution of cichlid fishes in Lake Bacalar, Yucatan Peninsula. Env. Biol. Fishes 54: 35-43.

Günther, A. 1867. On the fishes of the states of Central America, founded upon specimens collected in fresh and marine waters of various parts of that country by Messrs. Salvin and Godman and Capt. J. M. Dow. Proc. Zool. Soc. Lond.

Gyldenholm, A.O. \& J.J. Scheel. 1971. Chromosome numbers of fishes. I. J. Fish. Biol. 3: 479-486.

Hulsey, C.D. \& F. J. García De León. 2005. Cichlid jaw mechanics: linking morphology to feeding specialization. Funct. Ecol. 19: 487-494.

Hulsey, C.D., F.J. García De León, Y. Sánchez-Johnson, D.A. Hendrickson \& T.J. Near. 2004. Temporal diversification of Mesoamerican cichlid fishes across a major biogeographic boundary. Mol. Phylogenet. Evol. 31: 754-764.

Kligerman, A.D. \& S.E. Bloom. 1977. Rapid Chromosome preparations from solid tissues of fishes. J. Fish. Res. Board Can. 34: 266-269.

Levan, A., K. Fredga \& A.A. Sandberg. 1964. Nomenclature for centromeric position on chromosomes. Hereditas 52: $201-220$.

Martínez-Palacios, C.A. \& L.G. Ross. 1988. The feeding ecology of the Central American cichlid Cichlasoma urophthalmus (Günther). J. Fish Biol. 33: 665-670.

Martínez-Palacios, C.A. \& L. Ross. 1994. Biología y cultivo de la mojarra Latinoamericana Cichlasoma urophthalmus. CONACYT, México. D.F., México.

Martins, I.C., A.L.B. Portella-Castro \& H.F. Julio-Jr. 1995 Chromosome analysis of 5 species of the cichlidae family (Pisces-Perciformes) from Parana river. Cytologia 60: 223-231.

McKaye, K.R., J.R. Stauffer Jr., E.P. van den Berghe, R. Vivas, L.J. López-Pérez, J.K. McCrary, R.Waid, A. Konings,
W.J. Lee \& T.D. Kocher. 2002. Behavioral, morphological and genetic evidence of divergence of the midas cichlid species complex in two Nicaraguan crater lakes. Cuadernos de Investigación de la U.C.A. 12: 19-47.

Mendoza, A., S. Páramo-Delgadillo, J.A. Oseguera, C.A. Vázquez, J. Macias \& G. Bravo. 1992. Efecto de la densidad de carga y el nivel de proteína sobre el crecimiento de Cichlasoma syspilum (Pisces: Cichlidae) bajo cultivo experimental en sistema cerrado. Universidad y Ciencia 9: 53-63.

Mendoza, E.A., A. Mendoza, A. Galmiche \& R. Mesenguer. 1995. La acuicultura de peces nativos en México: Retos y perspectivas, p. 131-141. In E.A. Mendoza, A. Galmiche \& R. Mesenguer (eds.). Memorias del II Seminario sobre peces nativos con uso potencial en acuicultura. Colegio de postgraduados en ciencias agrícolas, Cárdenas, Tabasco, México D.F., México.

Michele, J.L. \& C.S. Takahashi. 1977. Comparative cytology of Tilapia rendalli and Geophagus brasiliensis (Cichlidae, Pisces). Cytologia 42: 535-537.

Miller, R.R. 1992. Pisces, p. 486-501. In S.H. Hurlbert \& A. Villalobos-Figueroa (eds.). Aquatic biota of México, Central America and the West Indies. San Diego, California, EEUU.

Moreira, F.O., A.S. Fenocchio, M.C. Pastori \& L.A.C. Bertollo. 2001. Occurrence of a metacentric macrochromosome B in different species of the genus Astyanax (Pisces, Characidae, Tetragonopterinae). Cytologia 66: 59-64.

Nishikawa, S., A. Kunio \& K. Tsuneo. 1973. A preliminary study on the chromosomes of Cichlasoma citrinella (Cichlidae, Pisces). Chromosome Inf. Serv. 14: 32-33.

Páramo-Delgadillo, S. 1984. Ictiofauna de Rió Gonzáles y lagunas adyacentes, Tabasco, México. Universidad y Ciencia 1: 5-19.

Páramo-Delgadillo, S. 1985. Determinación del sexo en algunos cíclidos de Tabasco, México. Universidad y Ciencia 2: 61-64.

Pérez-Sánchez, E., J.F. Muir \& L.G. Ross. 2005. Livelihood options for the coastal zone of Tabasco, Mexico. Aquat. Resour. Cult. Develop. 1: 91-108.

Portela-Castro, A.L.B, H.F. Júlio-Júnior \& P.B. Nishiyama. 2001. New occurrence of microchromosomes B in Moenkhausia sanctaefilomenae (Pisces, Characidae) from the Parana River of Brazil: analysis of the synaptonemal complex. Genetica 110: 277-283.

Rab, P., P. Liehman \& M. Prokes. 1983. Karyotype of Cichlasoma tetracanthum (Pisces, Cichlidae) from Cuba. Folia Zool. 32: 185-188. 
Reséndez-Medina, A. \& M.L. Salvadores-Baledón. 1983. Contribución al conocimiento de la biología del pejelagarto Lepisosteus tropicus (Gill) y la tenguayaca Petenia splendida Günther, del estado de Tabasco. Biótica 8: 413-426.

Salas, E. \& J. Boza. 1991. Citotaxonomía comparativa de tres especies de Cichlasoma (Pisces: Cichlidae) nativas de Costa Rica. Rev. Biol. Trop. 39: 219-224.

Salgado, S.M., E. Feldberg \& J.I.R. Porto. 1994. Estudos citogenéticos na familia Cichlidae (Perciformes, Labroidei) da Bacia Amazônica central, p. 47. En V. Simp. Citogenet. Evol. e Aplic. de Peixes Neotropicais, Botucatu, Brasil.

Thompson, K.W. 1979. Cytotaxonomy of 41 species of neotropical cichlidae. Copeia 4: 679-691.

Thorgaard, G.H. \& J.E. Disney. 1990. Chromosome preparation and analysis, p. 171-190. En C.B Schreck \& P.B. Moyle (eds.). Methods for fish biology. American Fisheries Society. Maryland, EEUU.
Uribe-Alcocer, M., L.B. Náder-Garcia \& N. ValdésMorales. 1992. Karyotypes of two Cichlid fishes from México, Cichlasoma ellioti and Cichlasoma trimaculatum. Jap. J. Ichthyol. 39: 174-177.

Uribe-Alcocer, M., C. Téllez-Vargas \& P. Díaz-Jaimes. 1999. Chromosomes of Cichlasoma istlanum (Perciformes: Cichlidae) and karyotype comparison of two presumed subspecies. Rev. Biol. Trop. 47: 1051-1059.

Valtierra-Vega, M.T. \& J.J. Schmitter-Soto. 2000. Hábitos alimentarios de las mojarras (Perciformes: Cichlidae) de la laguna Caobas, Quintana Roo, México. Rev. Biol. Trop. 44: 803-811.

Waltzek, T.B. \& P.C. Wainwright. 2003. Functional morphology of extreme Jaw protrusion in Neotropical cichlids. J. Morphol. 257: 96-106.

Zhang, Q. \& K. Arai. 2003. Extensive karyotype in somatic and meiotic cells of the loach Misgurnus anguillicaudatus (Pisces: Cobitidae). Folia Zool. 52: 423-429. 